\title{
Enthusiasm Adeptness in Wireless Position finder Multiuse building Via Envelope Severe Technique
}

\author{
Ms. Mahalakshmi. ${ }^{(1)}$, Dr. Dinesh Senduraja Ph.D. ${ }^{(2)}$ \\ Assistant Professor /Research Scholar (DIAT), Department of Computer Science, PKN Arts \& Science College, \\ Tirumangalam - 625706 . \\ Assistant Professor /Researcher (DRDO), Department of Computer Science, Pasumpon Muthuramalinga Thevar \\ College,Usilampatti - 625532.
}

\begin{abstract}
:-
The wireless instrument complex is an ad - hoc network. It consists of small light weighted wireless nodes called device nodes. The systematic model that allows us to derive some correct results vis-à-vis energy consumption and difficulty. Also, some main contemplations about the application of the proposed technique in a real instrument network, i.e., by taking into account erasure channels, MAC- layer overhead, and actual computational properties of nodes. The effect of important parameters such as nodes' concentration and program range through both extensive mockups and an methodical study of the trade-off between energy saving, convolution, and untrustworthiness of the proposed technique. A novel approach that splits the inventive messages into quite a lot of containers such that each node in the network will forward only small sub containers. The intense procedure is achieved applying the Packet Unbearable algorithm and the Chinese deposit proposition algorithm (CDP) which is characterized by a simple modular detachment between integers. The sink node, once all sub sachets is received correctly, will recombine them, thus reconstructing the original message. The excruciating process is especially helpful for those forwarding nodes that are more solicited than others due to their situation inside the network.
\end{abstract}

Keywords: MAC, CDS.

\section{Introduction}

In computer networking there is a great value of wireless schmoosing because it has no difficult connection, no more spending and has lots of ways to save ready band time. In the field of wireless schmoosing, there is another form of make contacts which is called as wireless instrument grid. A type of wireless schmoozing which is included of number of numerous devices and they are interlinked or coupled with each other for performing the same function together or cooperatively for the sake of examination and balancing the ecological factors. The wireless device network is an ad - hoc network. It consists of small light weighted wireless nodes called instrument nodes, deployed in fleshly or conservation condition. All sensor nodes in the wireless sensor network are interacting with each other or by intermediate sensor nodes. A instrument network consists of multiple detection stations called instrument nodes, each of which is small, lightweight and movable. Every instrument node is fortified with a transducer, microcomputer, transceiver and a power source. The transducer produces electrical indications based on

Sensed physical effects and phenomena.The microcomputer procedures and stores the instrument output. The transceiver, which can be hard-wired or wireless, receives guidelines from a central computer and transmits data to that computer. The power of each instrument node is resulting from the rechargeable utility or from a battery. Total working of wireless instrument schmoozing is based on its structure. The 
instrument grid initially involves of small or large nodes called as instrument nodes. These nodes vary in size and totally depend on the size because different sizes of instrument nodes work professionally in different fields. Wireless instrument schmoosing has such instrument nodes which are particularly designed in such a characteristic way that they have a microcontroller which controls the monitoring, a radio transceiver for producing radio waves, different type of wireless collaborating devices such as batteries.

\section{Factors affecting Throughput degradation in Optical Burst switching}

Optical Burst switching is the high speed switching technique that takes advantage of both Circuit and Packet switching. In OBS, the data bursts consisting of multiple data packets. When data packets arrive at an OBS node, data bursts are generated to carry the data packets to their destinations. For each newly generated data burst, a control packet is first sent to the destination of the data burst. The control packet reserves the resources at the intermediate nodes on the path of the data burst. No acknowledgment is sent back in order to minimize the delay time of sending out the data burst at the source. After an offset time, the source node sends out the data burst following the same routing path of the control packet. The minimum offset time between the control packet and the data burst is

$$
\text { Toff }=\mathrm{H} \times \mathrm{Tcp}+\mathrm{Tsw},(1)
$$

whereTsw is the required switch reconfiguration time at each node, Tcp is the processing time of a control packet in a node, $\mathrm{H}$ is the number of hops to the destination from the current location of the control packet. Hence, $\mathrm{H}$ is equal to the total hop count of the path when the control packet is at the source and decreases by one for each intermediate node the control packet passes.

One factor affecting performance of OBS is discarded-traffic clear approach. A data burst will be discarded if it cannot find an appropriate output channel when it arrives at an intermediate node. In this approach that discarded data burst cannot be retransmitted. This will consistently degrade throughput. Such problem can be avoided by using the discarded-traffic-retransmit approach.
Traditional OBS use First Come First Served approach to schedule the incoming data burst. This follows first in first out method. As each process becomes ready, it joins the ready queue. When the current running process ceases to execute, the oldest process in the Ready queue is selected for running. This increases delay that will degrade the performance.

Another factor is control overhead with unacceptable processing time (Tcp). Throughput mainly depends on control packet processing time. If Tcp increases, throughput decreases. Optical buffer is the only choice to introduce delay in between control packet and burst in order to compensate control packet processing time. Just Enough Time (JET) is one of the reservation schemes used in OBS. Here, the size of the data burst is decided before the control packet is transmitted by the source. If bandwidth is available, the control packet reserves channel for a fixed duration of time. The reservation is made from the time when the first bit of payload reaches the node till the last bit of payload is transmitted to the output port. Since, there is no wastage of bandwidth in this scheme, it removes idle bandwidth time. So we use JET scheme for all our performance evaluations.

\section{Container promoting system}

The container promoting system is the imparting of containers from one network section to another by nodes in a processor network. The meekest forwarding model unicasting involves a package being relayed from link tolink along a chain important from the packet's foundation to its endpoint. However, other forwarding approaches are usually used. Distribution requires a sachet to be reproduced and duplicates sent on many links with the goalmouth ofbringing a copy to every device on the grid. The redundancy espoused is in the form of manyduplicates of the same sachet that travel to the endpoint along several paths. However, manifold paths could unusually consume more energy than the single shortest path because several duplicates of the same containerhave to be sent.

\section{Container Dispensation}

The Container Dispensation refers to the wide diversity of algorithms that apply to a packet of data or evidence as it moves through the various network rudiments of a 
communications network. There are two broad classes of container dispensation algorithms that align with the consistent network sector of control flat and data flat. The algorithms are applied to either, Control information contained in a container and are used to transfer the container safely and professionally from cause to terminus The data content (regularly called the freight) of the container andare used to afford some satisfied

\section{Container Severe}

The Container Severe was the splitting of the containers into various sub-containers and to split the nodes and transmit containers towards the nodes. The original messages are riven into several containers such that each node in the complex will forward only small sub containers and restructure them back. The severe method is completed applying the Container severe Algorithm. And by this the wanted goals have beenrealized.

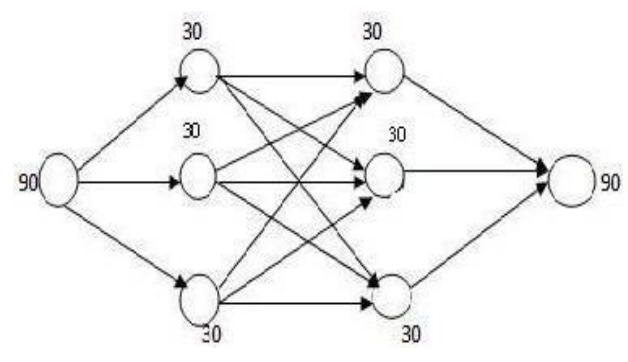

Fig 1 Container severe

The descend node, once all sub containers is received correctly, will recombine them, thus reconstructing the original message. The severe procedure is exclusively helpful for those progressing nodes that are more solicited than others due to their position inside the network. The original messages into several containers such that each node in the system will forward only small sub containers. The severe procedure is achieved applying the Container severe Algorithm. A thorough investigative model that allows us to derive some accurate results regarding energy feasting and complexity. Also, some main deliberations about the implementation of the projected technique in a real instrument system, i.e., by taking into explanation erasure stations, MAClayer upstairs, and authentic computational resources of nodes.Furthermore, the effect of important parameters such as nodes' density and show range through both general simulations and an systematic study of the tradeoff between vitality saving, complexity, and dependability of the proposed system.

\section{Cdp-based forwarding technique}

The CDP (Chinese Deposit Proposition) for Data sachet id, in which a node flinches at a arbitrary spot, then Applying Key Statistics to the Data containers for some Retreat Persistence. For these purpose intruders won't identify the data packet order. The Chinese Deposit Proposition is a result about similarity in quantity theory and its oversimplifications in intellectual algebra. In its basic form, the Chinese Deposit Proposition will regulate a number $\mathrm{n}$ that when separated by some given divisors leave given remainders. This module is mainly used for security persistence because it is vastly robust. The Chinese Deposit Proposition is a result about congruence's in number theory and its generalizations inintellectual algebra. In its basic form, the Chinese Deposit Proposition will govern a number $n$ that when alienated by some given divisors leave given residues. For example,what is the lowest number $n$ that when divided by 3 leaves a remainder of 2 , when divided by 5 leaves a remainder of 3 , and when divided by 7 leaves a remainder of 2? A common starting example is a woman who tells a policeman that she lost her basket of eggs, and that if shetook three at a time out of it, she was left with 2, if she tookfive at a time out of it she was left with 3 , and if she took seven at a time out of it she was left with 2. She then asks the policeman what is the minimum number of eggs she must have had. The answer to both problems is 23 .

\section{Container severe algorithm}

The Container Severe was the severe of the containers into various sub-containers and to divided the nodes and spread containers towards the nodes. The original messages are riven into several containers such that each node in the system will forward only small sub containers and reconstruct them back. This technique was achieved by using the container severe algorithm. Here we apply Key Statistics to the Data containers for some Sanctuary Resolve. For these resolution stalkers won't find the data container demand.

\section{Quantifiable}

In announcement systems, such as Ethernet or container radio, throughput or linkage throughput is the typical rate of successful 
message transfer over a letter channel. This data may be delivered over a corporeal or logical link, or pass through a certain network node. The throughput is usually unhurried in bits per second (bit/s or bps), and sometimes in data sachets per second or data containers per time slot. The organization throughput or combined throughput is the sum of the data rates that are delivered to all terminuses in a network. The throughput can be analyzed mathematically by means of queuing theory, where the load in containers per time unit is represented arrival rate $\lambda$, and the throughput in envelopes per time unit is denoted departure rate $\mu$.

\section{Container Carriage Section}

The container carriage ratio is definite as the ratio of data containers received by the terminuses to those produced from the sources. This recital metric gives us an idea of howwell the decorum is performed In terms of container carriage at unlike speeds using diverse transportation models. Mathematically.

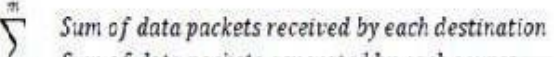 Sin of ate peikets gemeretel by each source \\ $\operatorname{PDR}(\%)=$ \\ $m$ \\ where $i$, indicates the number of output file \\ $\mathrm{m}$, indicates the total number of ouput files}

\section{End to End Delay}

End -to-end delay refers to the time taken for a packet to be transmitted across a network from source to destination.

dend-end $=\mathrm{N}[$ dtrans + dprop + dproc $]$

\footnotetext{
Where

dend-end $=$ end-to-end delaydtrans $=$ transmission delaydprop= propagation delay dproc $=$ processing

delay $\mathrm{N}=$ number of links (Number of router +1$)$ Note: we have neglected queuing delays.

Each router will have its own dtrans, dprop, dprochencethis formula gives a rough estimate.
}

\section{Controlled Overwhelming Burden}

Controlled Overwhelming Burden is defined as the total number of routing containers transferred per data container.

It is calculated by distributing the total number of beating containers showed (includes forwarded routing containers as well) by the overall number of data containers received.

\section{Systematic grades}

In this section, we derive some systematic grades regarding the projected CDP-based forwarding method. The main results are briefly brief as follows.

1) It is shown that by fixing the measurement of the packet, a maximum value of the number of CDP mechanisms, exists above which the dynamism drop factor starts to diminution. We elucidate the reason for this comportment and how to acquire this edge.

2) The influence of the amount of allowable bullets, on the ERF and on the network dependability Is assessed logically.

3) An investigative classical that can be used to estimation the mean oomph lessening factor possible with the Projected for- warding system is derivative, and it is verified that, under suitable environments, the planned Advancing algorithm is able to decrease the mean dynamism ingestion by about $37 \%$.

4) The above due to a conceivable MAC heading is systematically derived.

\section{Routine assessment}

In this section, we equate the concert of CDP in terms of energy consumption to those obtained by SP. Moreover, we deliver some fallouts obtained associating the CDP to the most naive severe scheme, a meek container detachment into chunks. The results have been obtained through a custom MATLAB simulator. We first show a assessment between the results obtained over the analysis and those found through the simulator. Then, we analyze some other parameters in order to show the advantages of the proposed technique. Let us consider a sensor network where nodes are randomly distributed in a square area of sizem , with density nodes/m. Instrument nodes are assumed to be static as usual in most application scenarios. In each simulation, the sink node is located in the center of the square grid, and each instrument node has a transmission range equal to $\mathrm{m}$. As designated in Section IV-D, the network is organized in clusters numbered in rising order starting from 
the cluster where is located the sink node, which is acknowledged with. We also assume that events randomly occur in faraway clusters such that. Simulations neglect the effect of collisions and retransmissions at the MAC layer. However, some results performed through the ns-2 simulator

[16] show that their impact on the values RS, $\mathrm{LT}$, and $\mathrm{Xu}$

Codes are those reported in by considering operations carried out on containers of one word each instead of a single container ofsub words.

\section{Table I Simulation ResultsObtained}

\begin{tabular}{|c|c|c|c|c|}
\hline MAC & Mechanism & Packets sent & Collisions & Average Delay \\
\hline \multirow{2}{*}{802.11} & CRT & 122.5 & 3654 & $4.4 \mathrm{~ms}$ \\
& SP & 306 & 790 & $4.2 \mathrm{~ms}$ \\
\hline \multirow{2}{*}{802.15 .4} & CRT & 1545 & 239 & $74.1 \mathrm{~ms}$ \\
& SP & 618 & 41 & $75.5 \mathrm{~ms}$ \\
\hline
\end{tabular}



Fig.6.1. ERF versus sorted topologies, with different values of overall delay can be considered of the same entity for thetwo different forwarding mechanisms

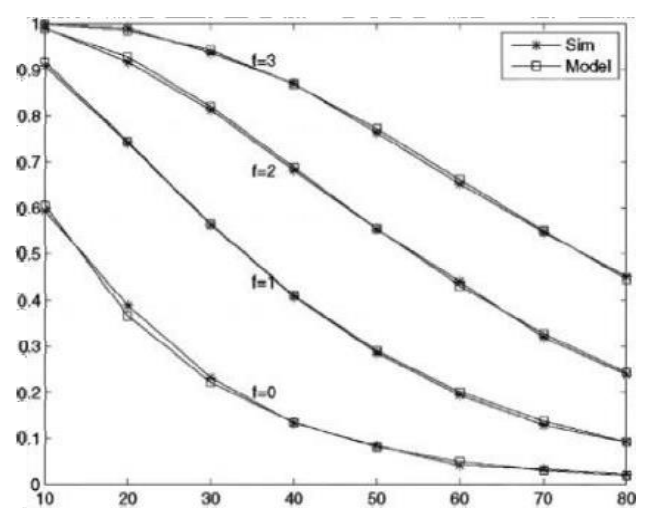

Fig.6.2. Comparison between the values calculated throughanalytical model and simulation

The simplest forwarding model involves a packet being relayed from link to link along a chain leading from the packet's source to its purpose. However, other forwarding strategies are commonly used. Propagation requires a packet to be repeated and copies sent on multiple links with the goal of distributing a copy to every maneuver to the sink node on the network. In practice, broadcast containers are not advanced everywhere on a network, but only to devices within a broadcast domain, making program a relative term. Less common than broadcasting, but perhaps of superior utility and theoretical meaning, is multicasting, where a containeris selectively duplicated and copies delivered to each of a set of recipients.

\section{Existing system}

A malicious third party (eavesdropper) retrieves sender's public component and sends his own public component to receiver. When receiver transmits his public key, third partyinterrupts and substitutes the value with his own public key and then sends it to sender. Now there is an agreement on a common secret key with third party instead of receiver. It is possible for third party (Man-in-Middle) to decrypt any messages sent out by sender or receiver

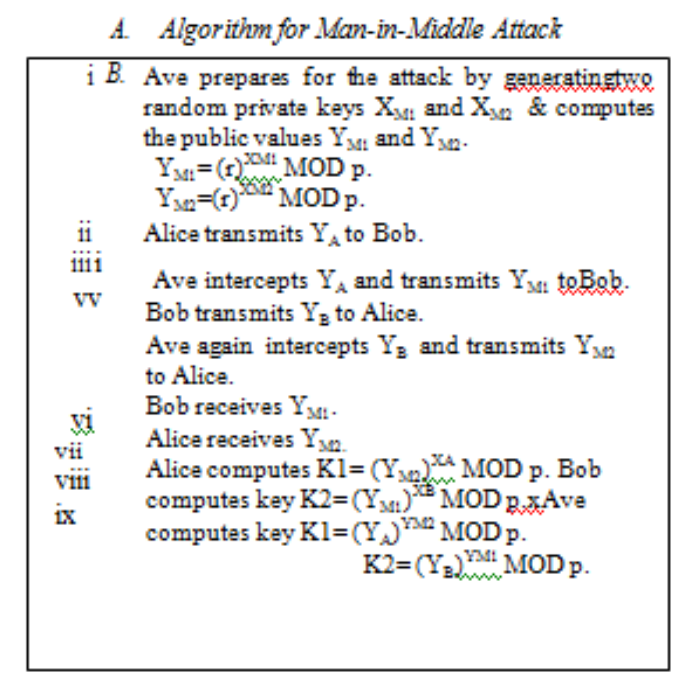

Fig. 1 Man-in-Middle Attack

Overcompensation of Tcp will increase the offset time between the control packet and the data burst when they pass the nodes along the path. Data bursts that have passed more nodes will therefore have a greater chance to reserve an output channel at an intermediate node because of the larger offset time. From implementation consideration, it may be better to first use FDLs to compensate the control packet processing time Tcp and then use other methods to further 
improve the throughput performance.



Fig. 2 Man-in-Middle Attack



Fig. 3 N party Man-in-MiddleAttack generation

\section{Implementation of proposedscheme and Result}

We have completed the research in following steps to getthe set objectives.

1. Deep study of Diffie-Hellman Key ExchangeProtocol.

2. Study of Man-in-the-Middle Attack Algorithm.

3. Proposed the algorithm for N-party Man-in-theMiddle Attack.

4. After shaping up of proposed algorithm, implement the algorithm in Ubuntu 14.04 usingPERL technology.

A. Proposed Algorithms

1) Algorithm at client end

1 Create a new socket if :-

i Assign peer host.

ii Assign peer port number.

iii Assign protocol Or Errormessage

2 Alice computes its own public componentand sends it to middle1.

i Take a prime number $\mathrm{p}$ and compute its primitive root $r$.

ii Alice take a private random number and computes public component. iii Send->\$socket(value)

3 Read the message sent by middle1 by using recv function.

\$socket->recv(value).

4Compute key by using received value.

Middle1 works not only as client but also as server.

It receives files from Alice as a receiver and sends to next useras a sender. So first we run server file and then clientfile atmiddle 1 end.

2) Algorithm at Middle1 end

1 Create a new socket if :-

i Assign peer host.

ii Assign peer port number.

iii Assign protocol or Error message

2 Wait for client connection.

3 When available, accept a new connection.

4 Middle 1 receives the data from Aliceuser.

5 Compute key.

6 Run client file at middle 1 end tocommunicate with next user.

i Create a socket and connect toserver.

ii Send data to next user.

iii Receive public component sentby middle 2 user.

iv Compute key

Middle2 works not only as client but also as server. It receives files from Middle1 as a receiver and sends to next user as a sender. So first we run server file and then client file at middle 2 end.

3) Algorithm at Middle2 end

1 Create a new socket if :-

i Assign peer host.

ii Assign peer port number.

iii Assign protocol Or Errormessage

2 Wait for client connection.

When available, accept a new connection

3 Middle2 receives the data fromMiddle1user.

4 Compute key.

5 Run client file at middle2 end tocommunicate with next user.

6 Create a socket and connect to server.

7 Send data to next user.

8 Receive public component sent by Bobuser.

9 Compute key

Bob works as a server. It receives files from Middle2 asa receiver and sends it to middle2. So we run server file at middle 2 end.

4) Algorithm at server end

1 Create a new socket if:-

i Assign peer host.

ii Assign peer port number.

iii Assign protocol Or Error message

2 Wait for client connection.

3 When available, accept a new connection.

4 Bob send own pubic key top middle2

5 Bob receives the data from Middle2 user.

6 Compute key.

B. Implementation and Result (Fig 4)

Alice key generation: - All random numbers less than 7 can be used as private value because we 
consider prime number7 to compute keys and we selected 3 as a primitive root of 7 . Alice generates random private values. When private valueis 3 then public component is computed as 6 and key is also 6 .

Table 1 Alice key generation

\begin{tabular}{|l|l|l|}
\hline Private randomvalues & Public components & Keys \\
\hline 3 & 6 & 6 \\
\hline 4 & 4 & 1 \\
\hline 6 & 1 & 1 \\
\hline 5 & 5 & 2 \\
\hline 2 & 2 & 4 \\
\hline 5 & 5 & 3 \\
\hline
\end{tabular}

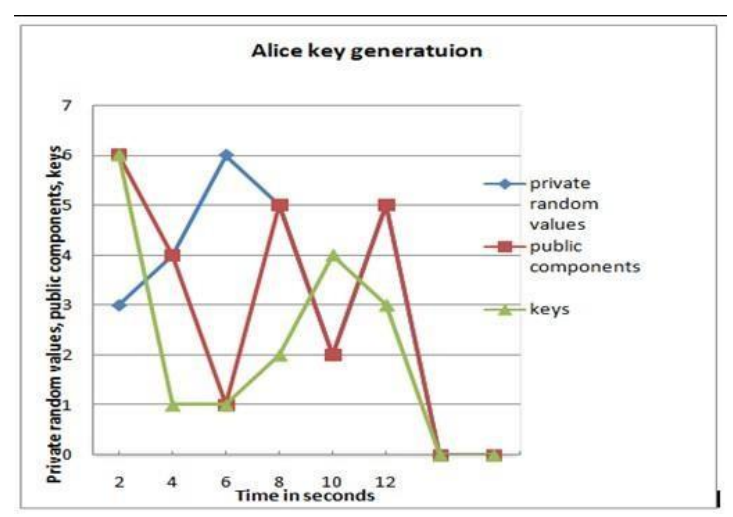

Fig. 5 Alice key generation

At specific time Alice generates random private values and computes public components. According to these values,keys are generated.

\section{Conclusion:}

In this paper, we have presented a novel forwarding technique for WSNs based on the Chinese Deposit Proposition (CDP). In particular, we have derived an logical model able to forecast the energy effectiveness of the method, and we have particularly focused on some implementation issues. First, we have discussed the choice The Diffie-Hellman key exchange protocol is very effective scheme to generate a common secret key for both the sender and the receiver. It is used mainly wherever we need to compute key for exchange the shared key.

It is not necessarythere is always one middle man; there may be many attackers between sender and receiver. When client sends his public component to server, first attacker intercept it and sends own generated first public components to server and second to client but if there is another attacker then that first component intercepted by second attacker.

In this type all attackers intercept public components of their neighbor users and generate their own keys which are similar to neighbor users and decrypt the messages. In our work, we generate $\mathrm{N}$ - party Man-in-the-Middle Attack in Diffie- Hellman key exchange protocol using PERL technology.

In the present work we considered 2-party manin-the- middle attack in Diffie-Hellman key exchange algorithm which can be extended to $\mathrm{N}$ party to create a network of intrusion. The main challenge will be to create a defense linefor $\mathrm{N}$ party attack and have a distributed detection systemfor such a scenario.

\section{References:}

[1] Akyildiz. I.F Su. W, Sankarasubramaniam .Y, and Cayirci. E (2012) "A survey on sensor networks" , IEEE vol. 40, no. 8, pp. 102- 114.

[2] Campobello .G, Leonardi. A, and S. Palazzo, (2018), "On the use of Chinese Remainder Theorem for energy saving in wireless sensor networks," IEEE May pp. 2723-2727.

[3] Campobello .G, A. Leonardi. A, and S. Palazzo, (2019), "A novel reliable and energy-saving forwarding technique for wireless sensor networks," IEEE(May 18-21, pp. 269-278.

[4] Cohen. K, (2011) “Energy-Efficient Detection in Wireless Sensor Networks UsingLikelihood Ratio and Channel State Information" IEEE vol. 43Pages:16711683.

[5] Ganesan. D, Govindan. R, Shenker. S, and Estrin. D, ," (2018) "Highly resilient, energy efficient multipath routing in wireless sensor networks" IEEE vol. 5, no. 4, pp. 10-24.

[6] [6] Hong. H. Wu. C. H, ( 2017) "RSA

[7] Cryptosystem based on the Chinese Remainder Theorem." IEEE vol. 5 pp. 391- 395.

[8] Jeong. Y.S, (2017) "Visualization of efficiency coverage and energy consumption of Sensorsin wireless sensor networks using heat map" IEEE vol 40 Pages:1129-1137.Jin-Shan Lee. (2018) "Fuzzy-Logic-Based Clustering Approach for Wireless Sensor Networks Using Energy Predication" IEEE Vol. 8 Pages: 2891-2897. 
[9] Otal. B, (2019), "Highly reliable energysaving mac for wireless body sensor networks in healthcare systems" IEEE vol.34 Pages:553-565

[10] Shunfu Jin, Wuyi Yue . (2018) "Performance evaluation of multi-traffic on wireless sensor networks using a novel Diffserv mechanism" IEEE Vol. 4 Pages: 377- 381.

[11] Swades De and Chunming Qiao (2018) "on throughput and load balancing of multipath routing in wireless networks" IEEE Vol. 3 page 1551- 1556.

[12] Zhiqiang Xiong, Zongkai Yang (2020) page (s): 1- 4, "A lightweight FEC algorithm for fault tolerant routing in wireless sensor networks" IEEE Vol 8 page (s): 1- 4,

[13] Lein Harn, Manish Mehta and Wen-Jung Hsin, "Integrating Diffie- Hellman Key Exchange into the Digital Signature Algorithm (DSA)", IEEE communications letters, vol. 8, no. 3, March 2004.

[14] Raphael C.-W. Phan, Member, "Fixing the Integrated Diffie-Hellman- DSA Key Exchange Protocol ", IEEE communications letters, vol. 9, no. 6, June 2005.

[15] Ik Rae Jeong, Jeong Ok Kwon, and Dong Hoon Lee, "Strong Diffie- Hellman-DSA Key Exchange", IEEE communications letters, vol. 11, no. 5, may 2007.

[16] Nan Li, "Research on Diffie-Hellman Key Exchange Protocol", 2nd International Conference on Computer Engineering and Technology, Vol 5, 2010.

[17] Barun Biswas, Krishnendu Basuli, "A novel process for key exchange avoiding man-in-middle attack", International Journal of Advancements in Research \& Technology, Volume 1, Issue 4, September2012.

[18] Barun Biswas, Krishnendu Basuli, Samar Sen Sarma, "On a key exchange technique, avoiding Man in the-middle Attack", Journal of Global Research in Computer Science Volume 3, September 2012. 